\title{
Ultrasonic Relative Positioning for Multi-Robot Systems
}

\author{
Frédéric Rivard, Jonathan Bisson, François Michaud and Dominic Létourneau
}

\begin{abstract}
Coordination of a group of mobile robots is facilitated when they are able to determine their positions relative to each other. Instead of using an absolute positioning approach with fixed beacons in the operating environment, we have developed a ultrasonic relative positioning system that allows each robot to perceive the distance and the angle of other nearby robots in relation to its own position. The system is based on time-of-flight evaluation of ultrasonic pulses and a RF communication link. The system has a precision of $8 \mathrm{~mm}$ and of $3^{\circ}$ over a $6.7 \mathrm{~m}$ range. This paper describes the system, its performance and its use on four Pioneer 2 robots moving in formation.
\end{abstract}

\section{INTRODUCTION}

For making a group of robots to reliably move in formation, coordinate their actions [1] or share information about their locations [2], they need to be able to localize each other. Using a fixed referential system (e.g., maps, GPS or ultrasonic beacons placed at known positions in the operating environment [3], [4], [5]) is possible but requires instrumenting or mapping the environment. Robots can determine where the others are by comparing positions expressed in relation to a common reference frame. Even if they do not sense their presence directly, they can indirectly determine the presence of others nearby.

When such engineering of the environment is not possible, a more appropriate approach is to have robots directly perceive the position (in distance and angle) of others in its surroundings. Humans do this all of the time using vision, and it is possible for mobile robots to do it too [6], [7], [8], [9], [10]. However, vision may lead to processing-expensive perceptual modules for reliable and omnidirectional sensing, compensating for the influence of varying illumination conditions, limited range of view and disambiguation of objects.

To overcome these limitations, we have developed a mobile ultrasonic relative positioning system (URPS) that can be used by robots to detect the distances and the angles of surrounding robots in relation to each other. Each robot is equipped with a device consisting of one transmitter, three receivers and one RF communication link [11]. The detection approach is based on time-of-flight evaluation of ultrasonic pulses. After describing the approach, the paper presents the

This research was supported financially by the Canada Research Chair, the Natural Sciences and Engineering Research Council of Canada and the Canadian Foundation for Innovation.

The authors are with the Department of Electrical Engineering and Computer Egineering, Université de Sherbrooke, 2500 boul. Université, Sherbrooke, Québec Canada Frederic.Rivard@USherbrooke.ca Jonathan.Bisson@USherbrooke.ca

Francois.Michaud@uSherbrooke.ca

Dominic.Letourneau@USherbrooke.ca implementation of the system and the results obtained using four Pioneer robots.

\section{ULTRASONIC RELATIVE POSITIONING SYSTEM}

Our system is based on time-of-flight evalutation of ultrasonic pulses from one transmitter (D) in relation to three receivers $(\mathrm{A}, \mathrm{B}, \mathrm{C})$ [4], [5], [12], as shown by Figure 1. instead of placing these receivers in known positions in the environment, our system mounts the receivers on the mobile robot so that the reference frame is the robot itself [11], [13]. The positions of the receivers in relation to the mobile robot are known. The device determines the position of a transmitter D located on another robot based on distance measurements returned by the receivers. The transmitter generates an ultrasonic pulse, and at the same time signals this emission to other ultrasonic relative positioning devices using the RF communication link. Since RF waves travel at the speed of light $\left(3 \times 10^{8} \mathrm{~m} / \mathrm{s}\right)$ and ultrasonic pulses travel at the speed of sound $v\left(343 \mathrm{~m} / \mathrm{s}\right.$ at $T=20^{\circ}$ Celsius $)$, the RF signal is detected by the receivers almost instantaneously. Using the time of flight $T O F$ detected by one receiver, the distance $d$ between the transmitter $j$ and the receiver $i$ can be found by multiplying it with the speed of sound, as expressed by Equation 1 . Using this equation to express $d_{A D}^{2}, d_{B D}^{2}$ and $d_{C D}^{2}$, the $\left(x_{D}, y_{D}\right)$ coordinates of the transmitter can be expressed by 2 .

$$
\begin{aligned}
d_{i j}= & T O F_{i j} \cdot v(T)=\sqrt{\left(x_{j}-x_{i}\right)^{2}+\left(y_{j}-y_{i}\right)^{2}} \\
& {\left[\begin{array}{ll}
2\left(x_{B}-x_{A}\right) & 2\left(y_{B}-y_{A}\right) \\
2\left(x_{C}-x_{A}\right) & 2\left(y_{C}-y_{A}\right)
\end{array}\right]\left[\begin{array}{c}
x_{D} \\
y_{D}
\end{array}\right]=} \\
& {\left[\begin{array}{c}
d_{A D}^{2}-d_{B D}^{2}+x_{B}^{2}-x_{A}^{2}+y_{B}^{2}-y_{A}^{2} \\
d_{A D}^{2}-d_{C D}^{2}+x_{C}^{2}-x_{A}^{2}+y_{C}^{2}-y_{A}^{2}
\end{array}\right] }
\end{aligned}
$$

Multipath propagation of ultrasonic pulses (caused by reflections) is not an issue here, since only the first detection (coming directly from the transmitter) is relevant. Accuracy is the biggest challenge with the device. Intuitively, increased accuracy can be obtained when the receivers are far away from each other, because delay measurements have a greater dynamic range. Before implementing the positioning device, we simulated it by placing three receivers at a radius $r_{i}=$ $100 \mathrm{~mm}$ (set according to Pioneer 2's dimensions) and $\theta=30^{\circ}, 150^{\circ}$ and $270^{\circ}$ around the origin. We evaluated in simulations two types of errors that could affect the accuracy of the device: absolute error which is common to all the receivers, and relative errors which are different for 


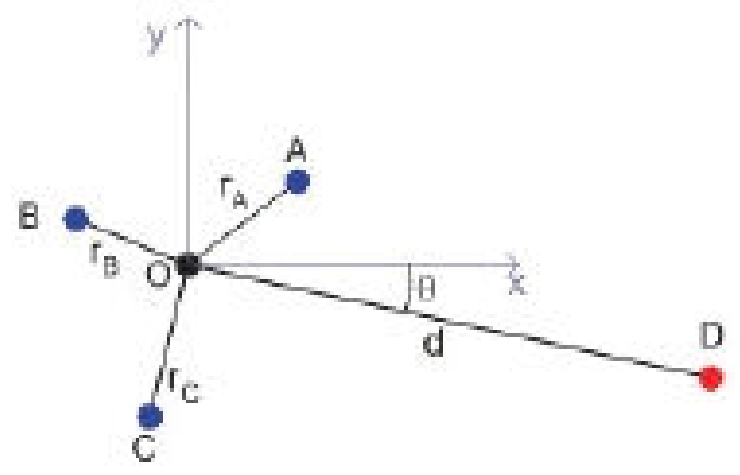

Fig. 1. Three fixed receivers (A, B, C) and one transmitter (D).

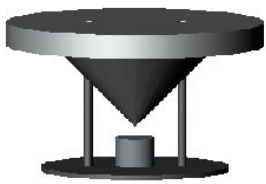

Fig. 2. Reflector cone on top of a transducer, making it omnidirectional.

each receivers. The absolute error can be introduced by the attenuation of the waveform, the processing required for the RF communication link or an error on the speed of sound used by the system. The relative errors can be caused by the small differences of the components of the receivers or the presence of objects surrounding the receivers. The simulations have shown that $d$ is affected by both types of errors while $\theta$ is mainly affected by relative errors [11], [13]. The resulting coordinates $\left(x_{D}, y_{D}\right)$ are very sensitive to noise on the measurements of TOF made by the receivers. Deriving both the distance $d$ and the angle $\theta$ from $\left(x_{D}, y_{D}\right)$ leads to a significant error on $d$, but gives a good precision on $\theta$. Thus, $\theta$ is derived using $\left(x_{D}, y_{D}\right)$ and $d$ is derived by taking the average over the three $T O F$ returned by receivers A, B and C. Distance measurements are also influenced by the difference in height between the transmitter and the receivers (a phenomenon that increases as the positioning devices are close to each other), but this can be corrected if this difference in height is constant and known. We also simulated the influence of $r$ (from $300 \mathrm{~mm}$ to 50 $\mathrm{mm}$, a physical limit for the size of the receivers) on the performance of the device. We observed that $r$ influenced mostly the angle measurements. In the worst case $(r=50$ $\mathrm{mm}$ ), the absolute mean error is around $10 \mathrm{~mm}$ and $5^{\circ}$. The accuracy on $\theta$ increases rapidly when receivers are distant: at $300 \mathrm{~mm}$, the absolute mean error is around $11 \mathrm{~mm}$ and $0.75^{\circ}$. The small increase on the distance error is caused by taking the average of TOF from the three receivers to derive $d$ : this approximation is valid only when the receivers are close to each other (i.e., when $r$ is small) [13].

To design such a system, ultrasonic transducers must be

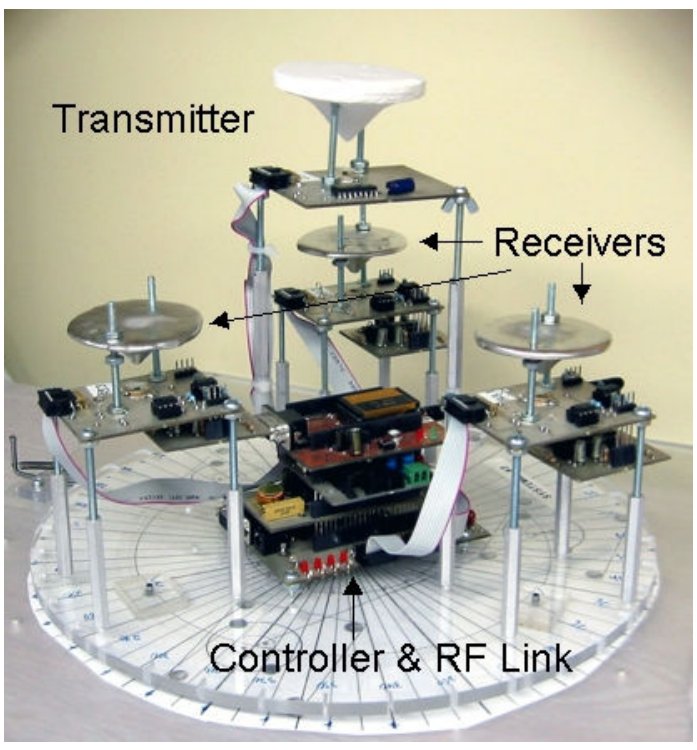

Fig. 3. Ultrasonic Relative Device

made omnidirectional. We decided to use a cone (see Figure 2) that reflects all incoming and outgoing ultrasonic pulses toward an ultrasonic transducer placed at the bottom [12]. We tested two types of cone, one made of plaster of Paris and the one made of lead, and both gave similar results. Tests were also conducted to observe the influence of the distance between the transducer and the tip of the cone: the bigger this distance is, the better the transmitted ultrasonic pulse is. We set this distance to $20 \mathrm{~mm}$.

Another design issue is the type of ultrasonic transducers to use. Electrostatic transducers operate on larger frequency band and range, and are more sensitive than piezoelectric transducers. Piezoelectric transducers are less expensive, smaller and require lower voltage levels, and so we chose to use them in our first prototype. Our first prototype [11], [13] used piezoelectric transducers at $40 \mathrm{kHz}$, but with those transducers interfered with Pioneer 2's ultrasonic range sensors (50 kHz electrostatic transducers). Our second prototype uses $25.7 \mathrm{kHz}$ piezoelectric transducers with a second order Tow-Thomas bandpass filter to prevent such interferences [14]. Figure 3 shows the resulting device. The transmitter is placed over the controller and higher than the three receivers, to avoid blocking incoming ultrasonic signals. We validated experimentally that the height difference is negligible and does not affect significantly the precision of the system. The RF communication link is a BIM2 transmitter/receiver at $433.92 \mathrm{MHz}$ from Radiometrix. The controller used is a PIC16F877 at $20 \mathrm{MHz}$ (5 MIPS). The microcontroller uses an internal counter to evaluate the time-of-flight of the ultrasonic pulse, giving it a maximum resolution of 4.2 us and a maximum value of $26.2 \mathrm{~ms}$ (resolution of $1.44 \mathrm{~mm}$ and maximum value of $d=9 \mathrm{~m}$ range at $343 \mathrm{~m} / \mathrm{s}$ ). Each ultrasonic relative positioning device consumes $1.73 \mathrm{~W}$ and operates at $7.5 \mathrm{~V}$.

For a group of robots using such devices, the overall 
system must synchronize the devices so that no more than one transmitter is active at all time. Each device is given a specific identification ID number (serving also as the ID number of the robot) and is set to transmit using round robin scheduling with a time watchdog (in case one device fails to communicate with the others) [14]. The refreshment rate of the system has been determined experimentally by identifying the minimum time between transmission of ultrasonic pulses from two devices. The ultrasonic pulse from one device must be attenuated enough before having triggering another device to avoid the previous pulse to be detected again. Equation 3 expresses this constraint, with $T_{g}$ being the group refreshment rate, $N$ is the number of robots in the group and $t_{\min }$ is the minimum time between transmission of ultrasonic pulses.

$$
T_{g s}=N \cdot t_{m i n}
$$

Tests were conducted in three different environments (open area, a corridor with gypsum walls and a corridor with concrete walls), with four robots placed at the corners of a $1 \mathrm{~m}^{2}$ (at a distance of $450 \mathrm{~mm}$ from the gypsum walls and $650 \mathrm{~mm}$ from the concrete walls). We tested the following set of $t_{m}$ in for each of these environments: 25, 50, 75, 100, 150 and $200 \mathrm{msec}$. When $t_{m}$ in is too small, erroneous data are returned by the ultrasonic relative positioning devices. Data are considered erroneous when the positions returned are greater than $\pm 200 \mathrm{~mm}$. Results are shown in Figure 4 . The minimal refreshment rate between two transmissions is $75 \mathrm{msec}$, and we set it to $100 \mathrm{msec}$ for the tests reported in the following section.

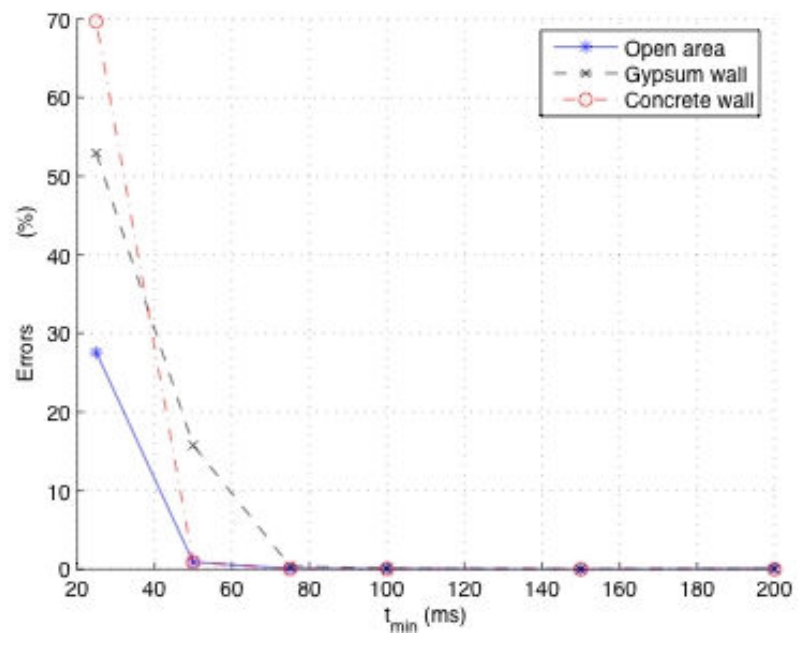

Fig. 4. Errors in relation of $t_{m i n}$.

\section{RESULTS}

The first set of tests consists in characterizing the capabilities of the system using the experimental setup shown in Figure 5. Placing a transmitter at specific positions (200 $\mathrm{mm}$ increments starting at $500 \mathrm{~mm}$, and $10^{\circ}$ increments),

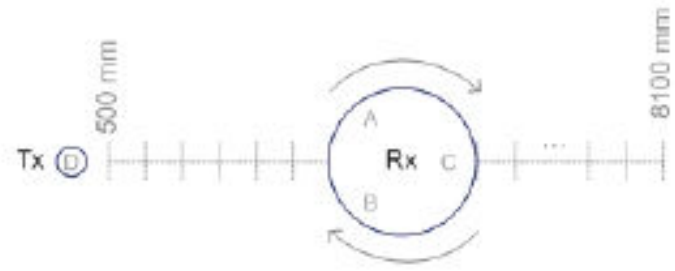

Fig. 5. Characterization of the ultrasonic relative positioning devices: Experimental setup.

TABLE I

CORRELATION RESULTS.

\begin{tabular}{|c|c||c|c||c|c||c|c|}
\hline \multicolumn{2}{|c||}{} & \multicolumn{2}{c||}{ Device 1 } & \multicolumn{2}{c||}{ Device 2 } & \multicolumn{2}{c|}{ Difference } \\
\cline { 2 - 8 } \multicolumn{2}{|c|}{} & $d(\mathrm{~mm})$ & $\theta\left({ }^{\circ}\right)$ & $d(\mathrm{~mm})$ & $\theta\left({ }^{\circ}\right)$ & $d(\mathrm{~mm})$ & $\%$ \\
\hline \hline \multirow{3}{*}{$2 \mathrm{~m}$} & Ave & 1996 & -1.3 & 1994 & 3.5 & 2 & 0.1 \\
\cline { 2 - 8 } & Std & 4 & 3 & 2 & 0.60 & 2 & 0.1 \\
\hline \hline \multirow{3}{*}{$3 \mathrm{~m}$} & Ave & 2982 & -2.2 & 3001 & 1.4 & 19 & 0.7 \\
\cline { 2 - 8 } & Std & 3 & 2 & 3 & 2 & 0 & 0 \\
\hline \hline \multirow{3}{*}{$4 \mathrm{~m}$} & Ave & 3981 & -2.8 & 4013 & -4.3 & 32 & 0.8 \\
\cline { 2 - 8 } & Std & 4 & 3 & 5 & 3.85 & 1 & 0.03 \\
\hline
\end{tabular}

data processed by the devices were compiled over $360^{\circ}$ and $6700 \mathrm{~mm}$ (the maximum observed perception range using transmitter equipped with a reflector cone $-8100 \mathrm{~mm}$ was observed using a transmitter oriented horizontally [11], [13]). For each position, 20 readings were taken. Figure 6 illustrates the results. The average error is always below $20 \mathrm{~mm}$ and $5^{\circ}$. Over the $6700 \mathrm{~mm}$ range, the absolute average error is $8 \mathrm{~mm}$ and $3^{\circ}$, with standard deviations of $8 \mathrm{~mm}$ and $3^{\circ}$ and absolute maximum errors of $69 \mathrm{~mm}$ and $17^{\circ}$.

We then conducted correlation tests using two devices placed face-to-face at three distances $(2 \mathrm{~m}, 3 \mathrm{~m}, 4 \mathrm{~m})$. For each distance, 1200 readings have been collected. The average readings and standard deviations are reported in Table I. Distance measurements should be correlated between the devices because they are facing each other. The difference between the angles are not necessarily correlated because they are influenced by the alignment of the robots. Stable readings are observed, and the distance error increases with the distances between the devices but always remains below $1 \%$ of the real distance between the two.

Finally, a third set of tests were conducted to evaluate the capabilities of the system to work in multi-robot conditions. Figure 7 shows a robot equipped with an ultrasonic relative positioning device. The devise is placed on an illuminated color cylinder, making it possible for the robots to localize each other using vision and the detection of colored blob in the robot's field of view. Visual positioning of robots has been used to develop and validate multi-robot formation [15] and platooning [16] approaches. Our objective in this set of tests was to see how the use of our ultrasonic relative positioning system (URPS) could be used to replace visual positioning in multi-robot formation trials, using Lemay et 

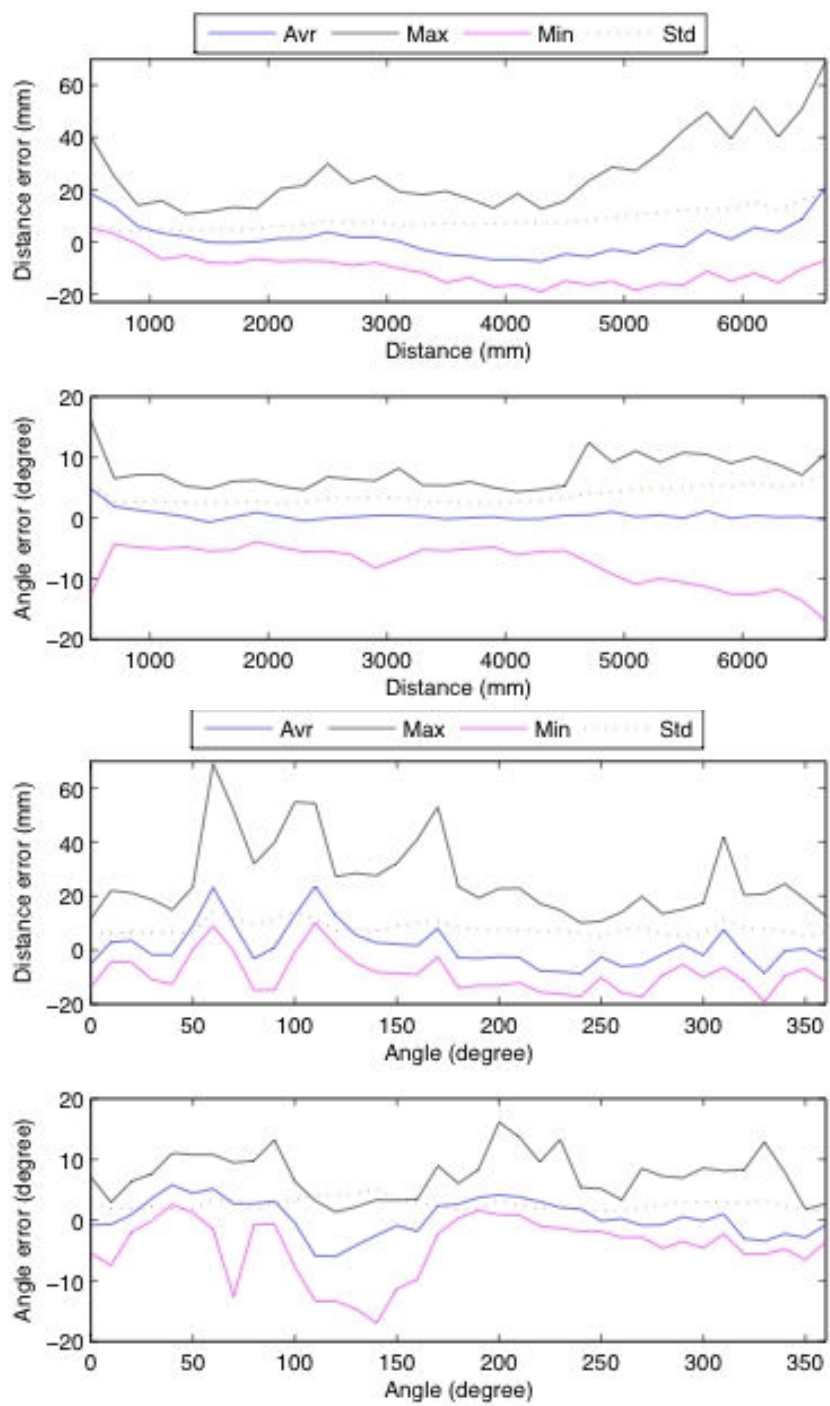

Fig. 6. Characterization of the ultrasonic relative positioning devices: Distance errors (top), and Angle errors (bottom).

al.'s [15], [17] distributed approach. This approach allows a group of robots to initialize their positions (by determining which robot to follow with the specific distance and angle) in relation to the desired formation and their initial poses, to then move in the desired formation.

Using a group of four robots, our ultrasonic relative positioning system was tested in four conditions, in a controlled environment with uniform illumination conditions:

- Initialization. Placed over a $4 \mathrm{~m} \times 4 \mathrm{~m}$, five sets of initial random positions were tested five time each. The objective is for the robots to initialize their positions by minimizing their displacement to form an arrow formation. These conditions allowed the ultrasonic relative positioning system to be used with robots placed at various distances and angles from each other. The robot's velocity was set to to $100 \mathrm{~mm} / \mathrm{sec}$. For each set of initial positions, the use of the ultrasonic relative positioning system provided constant and optimal positioning of

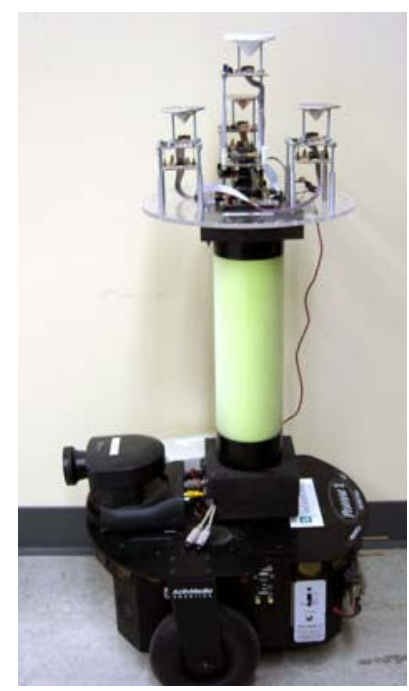

Fig. 7. Robot equipped with an ultrasonic relative positioning device.

the robots in the formation. In similar conditions, using vision for relative positioning of the robots produced stable results only $90.7 \%$ of the time. In addition, because directional cameras were used, each robot had to turn on itself to perceive the location of others in its surrounding. The ultrasonic relative positioning system, being omnidirectional, provided direct perception of its surroundings.

- Stability. When positioned in a formation, observing a stable formation would confirm that our ultrasonic relative positioning system provides reliable data. Three formation configurations (Arrow, Column, Diamond) were tested five times each over a $4 \mathrm{~m}$ range The distance between the robots is fixed to $1100 \mathrm{~mm}$, and a robot is considered not to be at its position when it is not within $275 \mathrm{~mm}(25 \%)$ or $15.75^{\circ}$ (35\%) of its desired relative position. Table II presents the percentage of time the robots were considered in position, for both the ultrasonic relative positioning system (URPS) and the vision relative positioning system. Stable and excellent performance are observed with the ultrasonic relative positioning system in all cases. The precision of the vision system is affected by the number of pixels detected in the color blobs perceived, which affects the precision of visual relative positioning even in good illumination conditions. The ultrasonic relative positioning system provides stable results over a wide range of positions.

- Switching. These trials involved having the robots switch formations (from Line to Arrow, Arrow to Column, and Column to Diamond) while moving. Reaching a stable and optimal (minimizing the displacement of each robot) configuration in the shortest distance traveled by the leading robot is the objective. Figure 8 shows the resulting maneuvers. Table III compares the distances traveled by the leading robot when URPS and the vision system are used. Again, improved per- 
TABLE II

TIME IN FORMATION

\begin{tabular}{|c||c|c||c|c||c|c|}
\hline \multicolumn{1}{|c||}{} & \multicolumn{2}{c||}{ Arrow } & \multicolumn{2}{c|}{ Column } & \multicolumn{2}{c|}{ Diamond } \\
\cline { 2 - 7 } & URPS & Vision & URPS & Vision & URPS & Vision \\
\hline \hline Trial 1 & 100 & 92.20 & 94.01 & 91.43 & 96.88 & 0 \\
\hline Trial 2 & 100 & 97.22 & 100 & 43.94 & 100 & 84.44 \\
\hline Trial 3 & 100 & 83.76 & 100 & 0 & 100 & 68.89 \\
\hline Trial 4 & 100 & 94.40 & 90.65 & 12.61 & 94.26 & 70.73 \\
\hline Trial 5 & 100 & 93.85 & 100 & 92.11 & 100 & 82.30 \\
\hline \hline Average & 100 & 92.3 & 96.93 & 48.02 & 98.23 & 61.27 \\
\hline
\end{tabular}

formance is clearly demonstrated by URPS.

TABLE III

DISTANCE TRAVELLED BY THE LEADER DURING THE SWITCH.

\begin{tabular}{|c||c|c|}
\hline \multicolumn{1}{|c||}{} & \multicolumn{2}{c|}{ Distance $(\mathrm{m})$} \\
\cline { 2 - 3 } & URPS & Vision \\
\hline \hline Line $\rightarrow$ Arrow & 1.5 & 2 \\
\hline Arrow $\rightarrow$ Column & 2.5 & 4 \\
\hline Column $\rightarrow$ Diamond & 3 & 4 \\
\hline
\end{tabular}
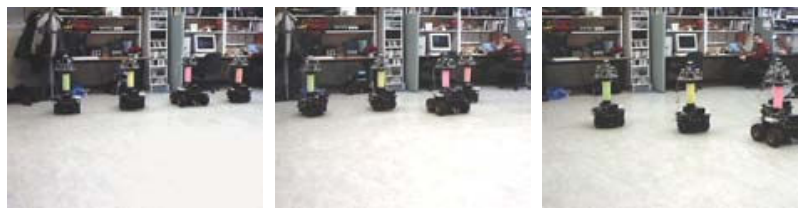

(a) Line to Arrow.
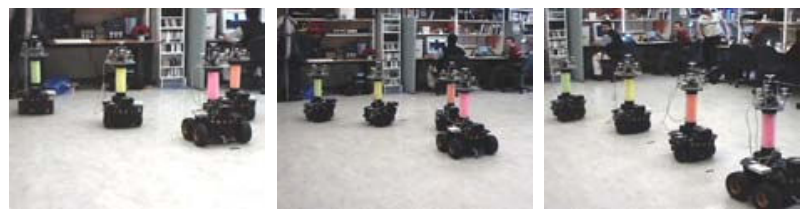

(b) Arrow to Column
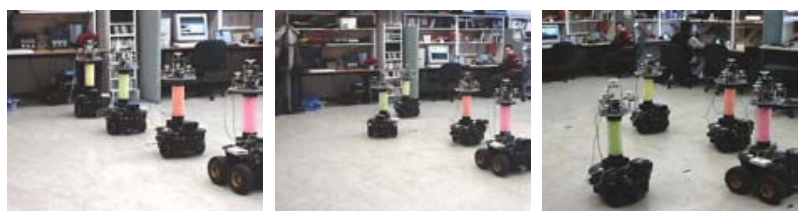

(c) Column to Diamond.

Fig. 8. Snapshots during switching maneuvers.

- Following a Human Leader. In this last test, we placed an ultrasonic transmitter on the experimenter, and observed if the robots were able to follow it in a Column formation. The formation successfully travelled multiple corridors and turned corners, as shown in Figure 9. This demonstration shows that the ultrasonic relative positioning system can be used outside the lab, in open conditions similar to what can be found in office buildings.

\section{RELATED WORK}

Known performance of fixed ultrasonic beacon systems are $2.5 \mathrm{~mm}$ of precision over usually $6 \mathrm{~m}^{3}$ for Figueroa and Mahajan's system [4], [5], and $29.3 \mathrm{~mm}$ and $1.84^{\circ}$ for $\mathrm{Wu}$ and Tsai's [18], in very specific test conditions. The small

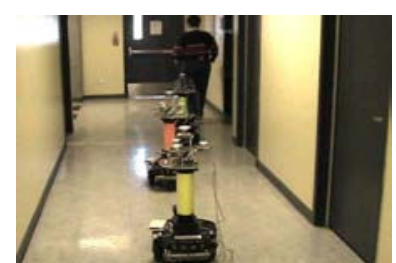

(a) First corner

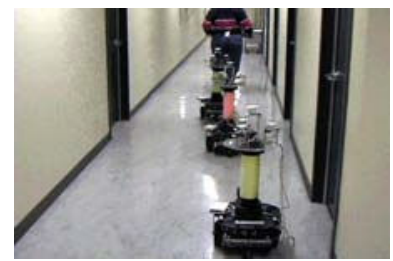

(c) Realignement.

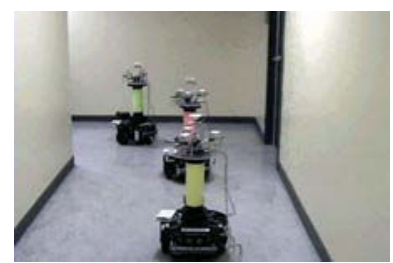

(e) The robots take a sharper turn.

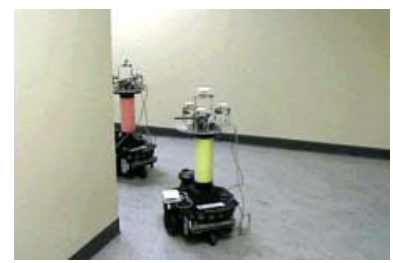

(g) Two robots passed.

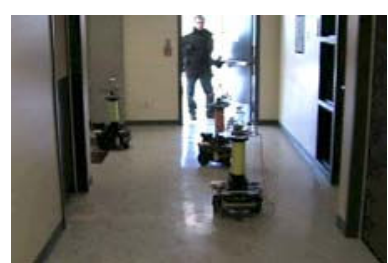

(b) The robots take the turn.

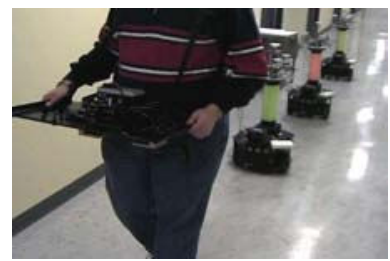

(d) Straight line.

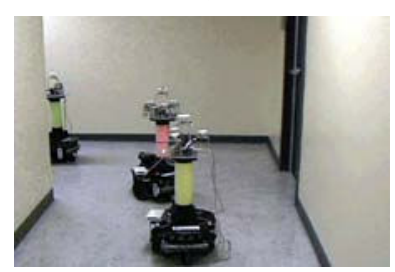

(f) First robot passed.

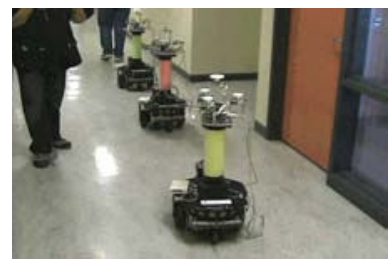

(h) Every robots passed.
Fig. 9. Group of three robots following a human in a corridor.

decrease in performance on our system comes with testing it in a more diverse set of conditions, and the added benefit of making the system portable directly on the robots. More recently, the Cricket system [19], [20] reports an accuracy of around 10 to $30 \mathrm{~mm}$ over a $1.2 \mathrm{~m}$ radius. The Millibots [12] robots use a similar system to ours. However, each robot is equipped with only one transducer, and so it can only determine the distance between one robot to another. To determine their relative angles, three Millibots must be used as fixed beacons, and one is set as the reference point. The overall accuracy is about $\pm 4 \mathrm{~mm}$, and the range is 3 m. Finally, the Parrots system [21] uses a similar approach to ours, but with devices equipped with four ultrasonic transmitters and four ultrasonic receivers placed on a square board. The accuracy is $20 \mathrm{~mm}$ over a $15 \mathrm{~m}$ range, but estimate the direction of the signal to approximately $90^{\circ}$.

Other sensors have been used for relative positioning. Premvuti and Wang [22] uses a rotating laser and photodiodes placed uniformly around the robot [22]. Knowing the rotational speed of the laser, the distance between two robots is determined by the detected speed of the laser beam emitted by the first robot, from the photodiodes of the other: the distance is proportional to the detected speed. The 
photodiode in the center of the detected beam correspond to the direction of the first robot in relation to the second. This system was validated in simulation only, and would be influenced by reflections. Kurazume and Hirose [23] uses a similar system, but only for positioning one robot. The SWARM project (www.irobot.com/rd/p07_Swarm.asp) makes a group of robots cooperate based on infrared signals. Each robot is surrounded by infrared detectors and emitters, and positioning can be infer from the detection of infrared signals all around each robot. Not much information is available on the system, but we can speculate that the precision of the system is determined by the number of infrared detectors around the robot. Kelly and Martinoli [24] presents and infrared-RF relative positioning system with $400 \mathrm{~mm}$ and $45^{\circ}$ accuracy over $3 \mathrm{~m}$. Arai et al. [25] also use infrared communication, but combined with an ultrasonic emitterreceiver. This approach requires more synchronization of the ultrasonic tranceivers between the robots, and its range is limited to $1 \mathrm{~m}$. SpotOn [26] localizes wireless devices relative to one another based on range estimated from radio signal attenuation, but no performance is provided yet.

\section{CONCLUSION}

Our omnidirectional ultrasonic relative positioning system allows a group of mobile robots to position themselves in relation to each other with an accuracy of about $8 \mathrm{~mm}$ and $3^{\circ}$ over a range of $6.7 \mathrm{~m}$, with a refreshment rate of $75 \mathrm{~ms}$ times the group size. Such performance is adequate for medium size robots such as Pioneer 2 operating indoors. Having the system deploy directly on the robots make it interesting for applications such as search and rescue missions in unvisited locations. For large number of robots, the group can be divided into subgroups networks to keep refreshment rate at an acceptable level. Fault tolerance strategies (e.g., in group settings, spurious detection can occur because of indirect reflection from a wall for robots that are not in direct lineof-sight) and data sharing (e.g., since one robot is positioned simultaneously by the rest of the group, cross-validation can be done by relaying position information from one robot to the others) can also be implemented using the system. In future work, we plan to improve the device by adding another receiver in order to determine position in 3-D instead of 2-D. Preliminary tests also indicated that the use of parabolic cones could improve the system's performance. A temperature sensor could also be added to adjust the speed of sound in real time.

\section{REFERENCES}

[1] R. Beckers, O. E. Holland, and J. L. Deneubourg, "From local actions to global tasks: stigmergy and collective robotics," in Proc. Artificial Life IV, R. A. Brooks and P. Maes, Eds. Cambridge, MA: The MIT Press, 1994, pp. 181-189.

[2] A. Howard, M. Mataric, and G. Sukhatme, "Cooperative relative localization for mobile robot teams: An egocentric approach," in MultiRobots Systems Workshop, 2003.

[3] H. R. Everett, Sensors for Mobile Robots. A.K. Peters, 1995.

[4] F. Figueroa and A. Mahajan, "A robust navigation system for autonomous vehicles using ultrasonics," Control Engineering Practice, vol. 2, no. 1, pp. 49-54, February 1992.
[5] _ - "A robust method to determine the coordinates of a wave source for 3-d position sensing," Journal of Dynamic Systems, Measurement, and Control, vol. 116, pp. 505-511, 1994.

[6] A. Das, R. Fierro, V. Kumar, J. Ostrowski, J. Spletzer, and C. Taylor, "A framework for vision-based formation control," IEEE Transactions on Robotics and Automation, 2002.

[7] J. Freslund and M. J. Mataric, "A general algorithm for robot formations using local sensing and minimal communications," IEEE Transactions on Robotics and Automation, vol. 18, no. 5, pp. 837846, 2002.

[8] F. Michaud, D. Ltourneau, M. Guilbert, and J.-M. Valin, "Dynamic robot formations using directional visual perception," in Proceedings IEEE/RSJ International Conference on Intelligent Robots and Systems, 2002, pp. 2740-2745.

[9] F. Michaud and M. T. Vu, "Managing robot autonomy and interactivity using motives and visual communication," in Proceedings International Conference on Autonomous Agents, May 1999, pp. 160-167.

[10] D. Fox, W. Burgard, H. Kruppa, and S. Thrun, "Collaborative multirobot localization," in Proc. of the German Conference on Artificial Intelligence (KI), Lecture Notes in Computer Science, vol. 1701, 1999, pp. 255-262.

[11] J. Bisson, F. Michaud, and D. Letourneau, "Relative positioning of mobile robots using ultrasounds," in Proceedings IEEE/RSJ International Conference on Intelligent Robots and Systems, 2003, pp. 1783-1788.

[12] L. E. Navaro-Serment, C. J. J. Paredis, and P. K. Khosla, "A beacon system for the localization of distributed robotic teams," in Proceedings International Conference on Field and Service Robotics, 1999.

[13] J. Bisson, "Localisation d'agents mobiles physiques," Master's thesis, Universite de Sherbrooke, Department of Electrical Engineering and Computer Engineering, 2003.

[14] F. Rivard, "Localisation relative de robots mobiles operant en groupe," Master's thesis, Universite de Sherbrooke, Department of Electrical Engineering and Computer Engineering, 2005.

[15] M. Lemay, F. Michaud, D. Letourneau, and J.-M. Valin, "Autonomous initialization of robot formations," in Proceedings IEEE International Conference on Robotics and Automation, 2004, pp. 3018-3023.

[16] F. Michaud, P. Lepage, P. Frenette, D. Letourneau, and N. Gaubert, "Coordinated maneuvering of automated vehicles in platoon," IEEE Transactions on Intelligent Transportation Systems, vol. 7, no. 4, pp. 437-447, 2006.

[17] M. Lemay, "Assignation dynamique de positions pour les deplacements en formation d'un groupe de robots," Master's thesis, Universite de Sherbrooke, Department of Electrical Engineering and Computer Engineering, 2005.

[18] C.-J. Wu and C.-C. Tsai, "Localization of an autonomous mobile robot based on ultrasonic sensory information," Journal of Intelligent and Robotic Systems, vol. 30, pp. 267-277, 2001.

[19] A. Smith, H. Balakrishnan, M. Goraczko, and N. Priyantha, "Tracking moving devices with the cricket location system," in Proceedings International Conference on Mobile Systems, Applications and Services, 2004.

[20] A. Priyantha and H. Balakrishman, "The cricket localtion support system," in Proceedings ACMIEEE International Conference on Mobile Computing and Networking, 2000.

[21] W. Zhang, J. Djugash, and S. Singh, "Parrots: A range measuring sensor network," Robotics Institute, Carnegie Mellon University, Tech. Rep. CMU-RI-TR-06-05, June 2006.

[22] S. Premvuti and J. Wang, "Relative position localizing system for multiple autonomous mobile robots in distributed robotic system system design and simulation," Robotics and Automation Systems, vol. 18, no. 3, pp. 319-326, 1996.

[23] R. Kurazume and S. Hirose, "An experimental study of a cooperative positioning system," Autonomous Robots, vol. 8, no. 2, pp. 43-52, 2000.

[24] I. Kelly and A. Martinoli, "A scalable, on-board localization and communication system for indoor multi-robot experiments," Sensor Review, vol. 24, no. 2, pp. 167-180, 2004.

[25] Y. Arai, H. Asama, H. Kaetsu, and I. Endo, "Distance measurment in multi-robot systems based on time shared scheduling," in Distributed Autonomous Robotic Systems, vol. 4, 2000, pp. 189-198.

[26] R. Hightower and G. Borriello, "Spoton: An indoor 3d location sensing technology based on rf signal strenght," University of Washington, Tech. Rep. UW-CSE-00-02-02, 2000. 